\title{
COVID-19 and allergy: how to take care of allergic patients during a pandemic?
}

\author{
Antonella Cianferoni ${ }^{1}$ \\ ${ }^{1}$ Children's Hospital of Philadelphia
}

August 12, 2020

\begin{abstract}
Severe acute respiratory syndrome coronavirus 2 (SARS-CoV-2), also known as COVID-19, is a new strain of coronavirus that has not been previously identified in humans. SARS-CoV-2 is recognized as a highly contagious respiratory virus with severe morbidity and mortality, especially in vulnerable populations. Being a novel disease, everyone is susceptible, there are no vaccine and no treatment. To contain the spread of the disease, health authorities throughout the world have restricted the social interactions of individuals in various degrees. Allergists like other physicians are faced with the challenge of providing care for their patients, while protecting themselves and patients from getting infected, with strategies that are in continuous evolution as States work through the different stages of social distance. Allergist provides care for patients with the most common noncommunicable disease in the world: asthma, allergic rhinitis, food allergy, venom allergy, drug allergy atopic dermatitis, and urticarial. Some of these diseases are not only considered risk factors for severe reactions but also have symptoms like cough and sneezing that are in differential diagnosis with COVID-19, and as we move forward may prevent allergy patient from working, go to school or access medical services that increasingly are allowing only asymptomatic patients. In this review, we will outline how to take care safety of different allergic patients during the pandemic.
\end{abstract}

\section{Introduction}

Severe acute respiratory syndrome coronavirus 2 (SARS-CoV-2), also known as COVID-19, is a new strain of coronavirus that has not been previously identified in humans and is thought to have originated in chrysanthemum bats in Wuhan City, Hubei province. ${ }^{1,2}$ Since December 2019, when Chinese public health authorities noticed several cases of acute respiratory syndrome in Wuhan City, SARS-CoV-2 outbreaks and clusters of the disease have since been observed in Asia, Europe, Australia, Africa, and the Americas and WHO declared a pandemic on March 11, 2020.

SARS-CoV-2 is now recognized as a highly contagious respiratory virus SARS-CoV-2 has multiple clinical presentations from asymptomatic to severe lung injury and multiorgan disease, especially in older individuals and those with chronic comorbidities. This polyhedric presentation makes it difficult to predict which health consequence the virus will have on the single individual and make it challenging to contain the spread..$^{3,4}$

Being a novel disease, everyone is susceptible, there are no vaccine and no treatment. To contain the spread of the disease, while developing treatment, vaccine, and hospital/health care preparedness, health authorities throughout the world have restricted social interactions of individuals in various degrees. Typically, the most restrictive measures are taken in the "red zone" or "Phase 1" when full quarantine measures are recommended for all citizens. In such a phase, only life-sustaining businesses are open, schools are closed, and there are government-imposed social distancing rules. These extreme mitigation strategies are followed by a progressive reopening approach with different phases like "orange zone", "yellow zone" "green zone" or phase 2,3 etc. that ease the restrictions as the virus becomes less prevalent in the community and health care systems are more prepared to treat the infected individuals (Figure 1). ${ }^{5}$ 
Allergists, like other physicians, face the challenge of providing care for their patients while protecting themselves and patients from getting infected. To achieve this goal, they use tactics that are in continuous evolution, adjusting work practices to State-mandated restriction, without clear guidelines but largely guided by fragmented recommendations given by local, national, and international organizations. ${ }^{6}$

Allergists provide care for patients with the most common non-communicable disease in the world: asthma, allergic rhinitis, food allergy, venom allergy, drug allergy atopic dermatitis, and urticarial. Some of these diseases are not only considered risk factors for severe reactions but also have symptoms, like cough and sneezing, that are in differential diagnosis with Covid-19. Taking care of the atopic patients is therefore essential not only to reduce severe outcomes if patients get infected with COVID-19, but also to prevent symptoms that may preclude allergy patients from working, go to school or access medical services if they are suspected of having COVID-19. To take care of those patients, allergists use procedures that require close contact with patients and can aerosolize the virus, and many therapeutic approaches that modulate the immune system. Risk and benefit for the single patients and the staff need to be carefully evaluated before doing them. ${ }^{6,7}$

\section{How to avoid infection in the office: Prevention and Control}

We know that SARS-Cov2 spread mainly from person to person, for interaction below 6 feet $(1.8 \mathrm{~m})$ via large droplets, produced when an infected person coughs, sneezes, or talks. Those droplets can land in the mouth, nose, or eyes of people nearby and can be inhaled. Symptomatic and asymptomatic individuals can spread the disease very easily and sustainably between people. The virus can also spread in other ways, from touching surfaces or objects, feces, from animals to people and from people to animals, but these are not thought to be the main ways the virus spreads. ${ }^{5}$

Information from the ongoing COVID-19 pandemic suggests that this virus is spreading more efficiently than influenza $\left(\mathrm{R}_{0}=1-2\right)$, but not as efficiently as measles $\left(\mathrm{R}_{0}=12-18\right)$, which is highly contagious. ${ }^{8}$ We know that way to reduce person to person spreads are: Maintain good social distance (about 6 feet); wash your hands often with soap and water. If soap and water are not available, use a hand sanitizer with at least $60 \%$ alcohol; Routinely clean and disinfect frequently touched surfaces. ${ }^{5}$ While organizing the office space, it is very important that when possible, staff will maintain 6 feet distance from each other, and also health care providers will maintain when possible such distance from patients. This can be achieved by redistributing desks, staggering patient influx in waiting rooms, limit the number of people coming with the patient (an adult should come alone when possible, children should be accompanied by 1 adult, and no siblings should not be allowed), avoid communal lunch or food sharing. ${ }^{9}$ Universal masking of patients and staff have also shown to reduce transmission. ${ }^{7}$ Furthermore, while the vaccine is not available, the CDC and ECDC have recommended that healthcare providers use of personal protective equipment (PPE) typical for standard, contact, and airborne precautions and with the use of eye protection. Therefore, to take care of COVID infected or potentially infected patients, gowns, gloves, and either an N95 respirator (in the USA) or FFP3/FFP2 (in Europe) plus face shield and goggles or a powered air-purifying respirator (PAPR) should be used. However, there is a worldwide shortage of N95 and PARP; therefore, allergists may need to use a face mask instead of an N95 respirator if one is not available.

To reduce the risk of exposure, the allergist can decide to visit only asymptomatic patients, by strict screening phone call prior to the visit. Patients should be asked if they have been exposed to COVID-19 infected individuals, if they have traveled to areas of high transmission, and if they have any of the typical symptoms (Figure 1). Telehealth can be used instead of an in-person visit. Furthermore, procedures like spirometry and nebulizers should be avoided. ${ }^{5}$

To reduce the spread of the virus, policies in the office should be present in the office to self-quarantine of asymptomatic healthcare providers that have been directly exposed to sick patients or in the community.

For certain periods allergist may be asked to reduce the visit to only essential ones, in order to reduce the physical contact among people as well to preserve PPE and resources. 
It is important to note that many allergy/immunology services are elective and can be managed without face-to-face interaction or deferred outright for short periods. Prioritizing certain services may be required when countries go in the red zone to prevent disease spread or based on the availability of adequate PPE for health care providers (Table 1). ${ }^{6,7}$

We will here review how to treat specific conditions in the allergy office.

\section{Procedure in the allergy office: how and when to perform them}

Skin testing : Skin testing may require prolonged contact with the patients, and there is a risk of fluid body contamination, especially in children that may cry or cough while doing the procedure.

In the red zone, such a procedure can be delayed as often information is not essential for the immediate treatment of the patient.

Skin tests can be resumed in yellow/orange zone (Phase 2/3) if appropriate PPE is available for health care providers like a mask, eye protection, gowns, and gloves. ${ }^{6,7}$

Food challenges : food challenges represent the gold standard for the diagnosis of food allergy, as in vitro and in vivo testing have not enough specificity and sensitivity to predict tolerance of food in patients with $\operatorname{IgE}$ and not $\operatorname{IgE}$ mediated food allergies. For not IgE mediated food allergies like food protein-induced enterocolitis (FPIES), no other tests exist besides food challenge.

Introduction of peanuts in patients sensitized to peanuts may prevent the development of food allergies (LEAP), food challenges or office maybe be required to introduce peanuts in patients at high risk of developing peanut allergies who have a positive IgE for peanuts.

Diagnostic food challenges maybe food challenges, can largely be delayed except for peanut challenge in an infant for peanut introduction due to LEAP protocol or for introducing a food critical for appropriate nutrition to avoid intervention like $\mathrm{G}$ tube or NG tube placement. ${ }^{6,7}$

In the yellow/orange phase, food challenge may be resumed starting for those patients who are avoiding foods like milk, egg, wheat, or who have multiple food restrictions to avoid malnutrition and expand diet to essential foods. As during food challenges prolonged contacted potential life resuscitation may be required PPE should be worn, and some hospital may require a negative swab from patients within 72 hours similar to those patients requiring procedures.

\section{Immunotherapy}

Allergen injectable immunotherapy helps to reduce symptoms of allergic rhinitis. For those patients on maintenance, immunotherapy is considered essential, but the administration can be spaced to up to 6 weeks to reduce health care facility visits during the pandemic; dose adjustment may be needed until a regular schedule can be resumed. For patients on build-up dosing for inhalant allergens, consider allowing for a longer period between injections (up to 14 days). Consideration of levelling off dosing in patients who are coming less often for immunotherapy may need to be individualized based on patient-specific characteristics and tolerance of immunotherapy. The risk of reactions to ITS is highest during the escalation phase, and the risk and benefits of such risk need to be considered. Consequently, during the red zone, the initiation of immunotherapy should be avoided. As restriction ease and PPE, emergency services become more available more can be offered to patients in terms of immunotherapy as a strategy to maintain allergic rhinitis under control. $^{15}$

For venom allergy immunotherapy is considered a life-saving treatment, there should be no change in service for initiation or build-up venom immunotherapy (VIT) of patients with a history of a systemic reaction to the venom, this is an essential service provided by allergists. Patients on maintenance VIT can be spaced to every 2-3 months if they have been on maintenance for at least a year. ${ }^{15}$

Sublingual and oral immunotherapy environmental allergies should not be initiated in red zone restrictions but can be continued at home. 
Food allergy immunotherapy visits for initiation and escalation could also be delayed, with patients maintaining current home dosing for those who have already been initiated. ${ }^{6,7}$ The decision on when and how to restart the immunotherapy for food should be taken based on the availability of PPE, community transmission levels, and stage of immunotherapy. If patients due to pandemic have been maintained on a high dose and are 1-2 doses from maintenance, those patients are likely low risk and can be restarted first, when conditions ease. Patients at low risk of reaction (higher doses of oral immunotherapy, never reacted before) should be restarted first followed by those patients at increased risk of reactions as conditions improve.

\section{Spirometry}

Spirometry has the possibility of causing aerosolization, so special consideration needs to be made (McGowan, A et al Recommendation from ERS Group 9.1 (Respiratory function technologists /Scientists-Lung function testing during COVID-19 pandemic and beyond).

The European Respiratory Society (ERS) does not recommend any patients with symptoms of COVID-19 or flu-like symptoms are tested with spirometry or Lung Function Tests (LFTs) under any circumstances at this time. They recommend postponing all routine testing during the pandemic phase. COVID-19 patients must not be tested for a minimum of 30 days post-infection.

Full operation of lung function services may only resume when viral prevalence is low and reliable testing based on a combination of symptom screening, and testing is readily available.

In the interim period, ERS is recommended only for critical need and if these mitigation strategies can be implemented

A test should always be carried out with a high specification disposable in-line bacterial and viral

filter in place with minimum proven efficiency for the high expiratory flow of 600 to $700 \mathrm{~L} / \mathrm{min}$.

The use of disposable combined mouthpieces/sensors is not recommended at this time. Maximize the use of single-use consumables and dispose of the items with care e.g., nose clips, rubber mouthpieces, etc.

For highest risk patients requiring essential testing, lung function should be carried out in a negative pressure room and using equipment only for high risk or infected patients.

This may only be available at specialist centers.

We recommend the use of either FFP3 masks or FFP2, where the former is not available. The

duration of the use of protective masks should comply with local policy. Eye protection must always be worn e.g., goggles or face shields.

Disposable gloves should be used at all times when testing. These should be discarded afterward.

Exercise testing, nebulization, bronchial challenge tests, and other aerosol-generating

procedures should be postponed during the pandemic until the green phase is reached.

\section{Asthma}

Asthma is one of the most common lung diseases globally, and its incidence is still increasing in developing country. ${ }^{10}$ It is listed as a risk factor for severe disease by the CDC in the case of COVID-19 infection. ${ }^{5}$ This recommendation is largely based on the fact that SARS-CoV-2 is a coronavirus and can cause asthma exacerbation. However, other previous severe pandemic coronaviruses (SARS-CoV and MERS-CoV) have not been associated with asthma. Similarly, during this current pandemic, there are little data to demonstrate a specific increased risk for COVID-19 from asthma. Data from China, Korea, and the US note that asthma is not a strong risk factor for severe COVID-19 disease, and actually, severe patients appear to be less likely to have asthma or COPD than the general population..$^{9,11-13}$ However, these data are based on hospitalized patients and may have significant limitations due to selection and reporting bias. It is also possible that asthma may use more caution and increased social distancing to avoid infections. Nonetheless, there is no 
evidence that anti-inflammatory medication taken regularly to prevent inflammatory diseases are putting patients at increased risk of severe COVID-19 related disease. ${ }^{13,14}$ On the other hand, we know that patients with asthma during the pandemic continue to have a risk of exacerbation not only related to SARS-CoV-2 but also to other infectious and allergy triggers with well-known morbidity and mortality. Asthma exacerbation requiring medical emergency treatment may increase the risk of exposure to COVID-19 due to visits to health care facilities. ${ }^{6,7}$ Unless new evidence emerges that show otherwise, the current recommendations are to continue to follow guidelines to treat asthma, including the use of biologic agents - IL-5, IL-4/IL-13, and IgE ${ }^{6,7}$ Biologics are considered among the essential services that allergists need to continue to provide during a red zone social distancing rule. However, given the unknown consequences of immunomodulation with biologics, during COVID-19 infection, biologics should be paused until recovery in SARS-CoV-2 positive patients. ${ }^{15}$

Special consideration to treat asthma is to reduce or avoid all together the use of nebulizer and spirometry for their risk to cause aerosolization of the virus in case of patients are carries as per before discussion. ${ }^{6,7}$

Allergic Rhinitis

Similar to patients with asthma, the medical treatment of patients with allergic rhinitis should continue. ${ }^{6,7}$ No evidence put this category of patients at risk of severe complication; during a COVID-19 infection, not controlled allergic rhinitis in an individual with uncontrolled allergic rhinitis may increase the spread of diasease. ${ }^{6,7}$ As restrictive measures get eased allergic rhinitis patients if symptomatic may be prevented from accessing health care facilities, school, camp, and work as COVID-19 symptoms may overlap with allergic rhinitis and prescreening of symptomatic patients will be widely implemented. ${ }^{6}{ }^{7}$ Under red-zone circumstances, allergic rhinitis should be evaluated via telemedicine or telephone instead of in person. Evaluation for allergy can be postponed during red-zone/phase 1 restrictions. Performing skin testing or in vitro serum specific IgE testing to inhalants may be appropriate as countries go to yellow, orange zones if the risk of acquiring COVID-19 in health care facilities is low and adequate PPE protections are available to healthcare providers for the close contacts required to perform skin test. ${ }^{6,7}$

Allergen immunotherapy can be considered the following recommendation outlined before $1{ }^{16}$

\section{Food Allergy, Eosinophilic Esophagitis (EoE), Drug Allergy, and Anaphylaxis}

Food allergy is a group of various diseases that pose different risk factors for patients. Acute IgE mediated food allergy and food protein enterocolitis (FPIES) can be associated with the risk of acute life-threatening or otherwise severe reaction, eosinophilic esophagitis is linked with the risk of irreversible fibrosis. Many patients with food allergies are generally controlled if they avoid the known food that causes an issue. Delayed or deferred treatment in short to intermediate-term (a few weeks to even a few months) may not change the outcome of these diseases ${ }^{6,7}$.

Follow up can be delayed or done via telehealth.

Food challenges can largely be delayed except for peanut challenge in infants for peanut introduction due to LEAP protocol or for introducing a food critical for appropriate nutrition to avoid intervention like G tube or NG tube placement.

During Pandemic treatment for Anaphylaxis and FPIEs have also been slightly modified to avoid exposures to COVID-19 in the healthcare setting as those risk and reduced with control of the infection normal. Patients are advised during anaphylaxis episode to self-administer epinephrine and to call 911 only if symptoms do not resolve or worsen after first epinephrine dose; otherwise, they are advised to stay home. ${ }^{17}$

Similarly, patients are advised to treat FPIEs acute episode at home unless severe dehydration ensue. ${ }^{18}$

One challenge during pandemic is to find food allergen-free or of a particular brand that patients are accustomed to, therefore referral to nutrition to address the nutritional needs of patients may be warranted. ${ }^{19}$

Allergic Skin Disorders, drug allergy and venom allergy 
For allergic skin disorder specific recommendations on service reduction.

In patients with urticaria, angioedema, and atopic dermatitis, the majority of visits can be considered under the non-urgent category where face-to-face care can be postponed or conducted via phone with digital photos triage or telehealth. ${ }^{5,15}$

Patients who are doing biologics for any of the above diseases can continue to receive those during the pandemic. For patients with known hereditary angioedema who develop an acute episode, visit local urgent or emergency health care facilities is appropriate. Also, every effort should be made to obtain on-demand therapy for home administration. ${ }^{6,7}$

All visits can be postponed desensitization in the hospital setting for essential antibiotics can be done..$^{2,16}$

For venom allergy, being VIT of patients with a history of a systemic reaction to the venom, as this is a life-threatening condition, is considered an essential service, as discussed before. ${ }^{16}$

\section{Immunodeficiency}

Patients with Immunodeficiency are a high risk for complication for COVID-19, so balance for prompt face in face service and risk that those services pose need to be carefully evaluated. Those patients can be continued on IVIG per regular therapy, although the IVIG products are unlikely to cover for the current virus, given the still low prevalence in the general population. Consideration to switch to subcutaneous vs. IV formulation should be considered as well as at home vs. in hospital/office administration. Telehealth can be continued for those encounters that do not require in-person visit. ${ }^{6,7}$

\section{Conclusions}

A pandemic response is likely and once in a lifetime event, making challenging to reorganize clinical practice and continue to adapt those changes based on viral infection level in the community.

Any suggestions may, therefore, change rapidly based on the evolving situation on the territory. Limiting in-person visit may have significant financial consequences and also at a certain point delay of procedure may represent a more significant risk for patient than the potential risk of being exposed to COVID-19 and should be taken under consideration.

Table 1: patient management during "red zone" shelter in place

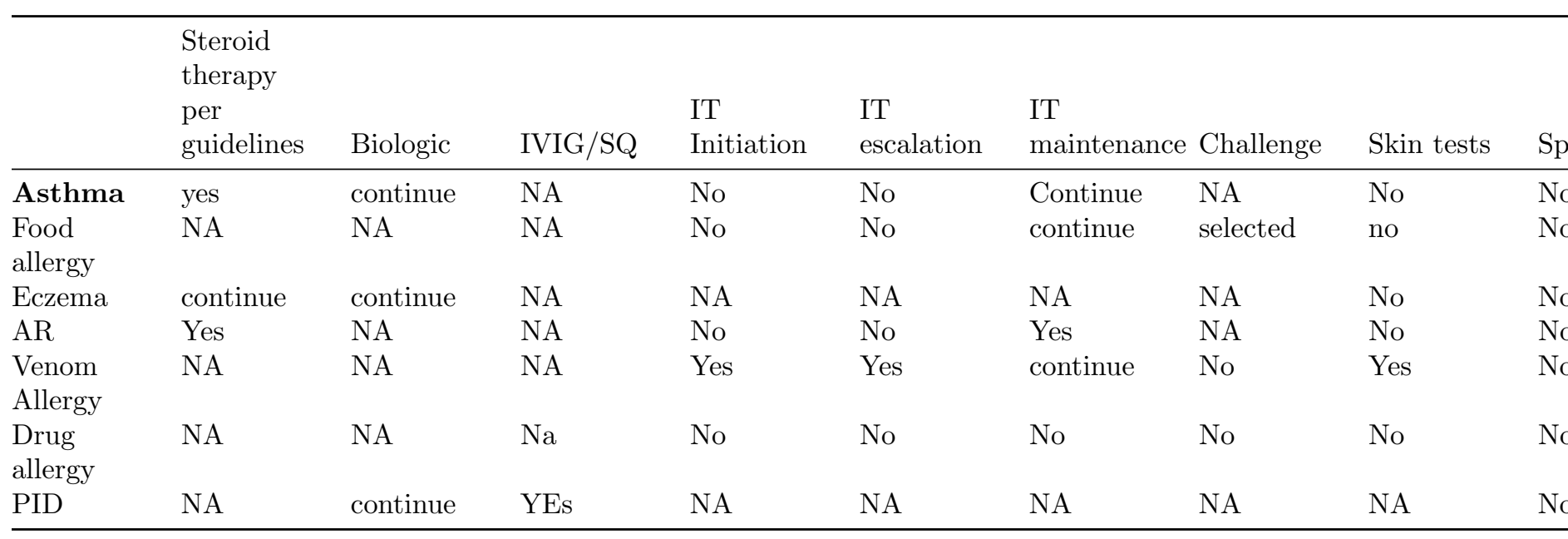

$\mathrm{AR}=$ allergic rhinitis; IT=immunotherapy; IVIG=intravenous immunoglobulin or subcutaneous IG; NA=not available; PID=primary Immunodeficiency

Table 2: Summary of Prevention Control measure in the office 


\begin{tabular}{ll}
\hline Prevention Measures & Implementation \\
\hline 6 feet $(1.8 \mathrm{~m})$ apart & Sign to remind to keep distance \\
& Reduce Number of staff and patients present at any given time \\
& Reduce or eliminate communal meals among staff, patients can not eating in office \\
PPE & Universal masking for staff and patients \\
& Standard, contact and droplet precaution \\
Wash hand & Wash hands for 20 second \\
Clean frequently high surface areas & Use alcohol at least $60 \%$ if washing is not possible \\
& Clean using manufacturing recs, look time of contact fpr disinfectant to clean areas \\
\hline
\end{tabular}

Figure 1: Viral community prevalence and safety recommendations

Figure 2: Questions to screen patients

\section{References}

1. Wu Z, McGoogan JM. Characteristics of and Important Lessons From the Coronavirus Disease 2019 (COVID-19) Outbreak in China: Summary of a Report of 72314 Cases From the Chinese Center for Disease Control and Prevention. JAMA 2020.

2. Ren LL, Wang YM, Wu ZQ, et al. Identification of a novel coronavirus causing severe pneumonia in human: a descriptive study. Chin Med J (Engl) 2020;133:1015-24.

3. Del Rio C, Malani PN. 2019 Novel Coronavirus-Important Information for Clinicians. JAMA 2020.

4. Bai Y, Yao L, Wei T, et al. Presumed Asymptomatic Carrier Transmission of COVID-19. JAMA 2020.

5. https://www.cdc.gov/coronavirus/2019-ncov/index.html. CfDCaPCDC-SSAf.

6. Shaker MS, Oppenheimer J, Grayson M, et al. COVID-19: Pandemic Contingency Planning for the Allergy and Immunology Clinic. J Allergy Clin Immunol Pract 2020;8:1477-88 e5.

7. Brough HA, Kalayci O, Sediva A, et al. Managing childhood allergies and immunodeficiencies during respiratory virus epidemics - the 2020 COVID-19 pandemic. Pediatr Allergy Immunol 2020.

8. Guerra FM, Bolotin S, Lim G, et al. The basic reproduction number (R0) of measles: a systematic review. Lancet Infect Dis 2017;17:e420-e8.

9. Guan WJ, Ni ZY, Hu Y, et al. Clinical Characteristics of Coronavirus Disease 2019 in China. N Engl J Med 2020;382:1708-20.

10. Dharmage SC, Perret JL, Custovic A. Epidemiology of Asthma in Children and Adults. Front Pediatr 2019;7:246.

11. Zhang JJ, Dong X, Cao YY, et al. Clinical characteristics of 140 patients infected with SARS-CoV-2 in Wuhan, China. Allergy 2020.

12. Korean Society of Infectious D, Korean Society of Pediatric Infectious D, Korean Society of E, et al. Report on the Epidemiological Features of Coronavirus Disease 2019 (COVID-19) Outbreak in the Republic of Korea from January 19 to March 2, 2020. J Korean Med Sci 2020;35:e112.

13. Haberman R, Axelrad J, Chen A, et al. Covid-19 in Immune-Mediated Inflammatory Diseases - Case Series from New York. N Engl J Med 2020.

14. Licari A, Votto M, Brambilla I, Castagnoli R, Piccotti E, Olcese R, et al. Allergy and asthma in children and adolescents during the COVID outbreak: What we know and how we could prevent allergy and asthma flares. Allergy. 2020 May 17. doi: 10.1111/all.14369.

15. Vultaggio A. Considerations on Biologicals for Patients with allergic disease in times of the COVID-19 pandemic. Allergy 2020;in press.

16. Epstein T. ADMINISTRATION OF SUBCUTANEOUS ALLERGEN IMMUNOTHERAPY DURING THE COVID-19 OUTBREAK: A Work Group Report of the AAAAI Immunotherapy, Allergen Standardization and Allergy Diagnostics (IASAD) Committee. 2020.

17. Casale TB, Wang J, Nowak-Wegrzyn A. Acute At Home Management of Anaphylaxis During the Covid-19 Pandemic. J Allergy Clin Immunol Pract 2020. 
18. Nowak-Wegrzyn A, Cianferoni A, Bird JA, Fiocchi A, Caubet JC, Medical Advisory Board of the International FA. Managing food protein-induced enterocolitis syndrome during the coronavirus disease 2019 pandemic: Expert recommendations. Ann Allergy Asthma Immunol 2020.

19. Groetch M, Durban R, Meyer R, Venter C, Nowak-Wegrzyn A. Dietary Management of Food ProteinInduced Enterocolitis Syndrome during COVID-19 Pandemic. Ann Allergy Asthma Immunol 2020. 